\title{
Estrutura Diamétrica e Arranjo Espacial das Espécies Mais Abundantes de um Fragmento de Floresta Estacional Semidecidual em Botucatu, SP
}

\author{
Luiz Alberto Blanco Jorge ${ }^{1}$, Thais Maria Millani ${ }^{1}$, Renata Cristina Batista Fonseca ${ }^{1}$, \\ Aparecido Agostinho Arruda ${ }^{1}$
}

${ }^{1}$ Departamento de Ciência Florestal, Faculdade de Ciências Agronômicas, Universidade Estadual Paulista - UNESP, Botucatu/SP, Brasil

\begin{abstract}
RESUMO
Foi feito um estudo sobre a estrutura diamétrica e o arranjo espacial das espécies mais abundantes que ocorrem em um dos cinco fragmentos florestais da Fazenda Experimental Edgardia (1.200 ha), município de Botucatu, SP. A área do remanescente é de 56 ha. Inventariaram-se 185 unidades amostrais contíguas de $100 \mathrm{~m}^{2}$, que compuseram uma amostra de 1,85 ha. Levantou-se a composição florística e estrutura horizontal da vegetação do centro do remanescente. Dentre as dez espécies de maior valor de importância, sete apresentaram abundância que permitiu realizar os estudos sobre distribuição diamétrica e distribuição espacial. O somatório dos índices de valores de importância (IVI) obtidos para as espécies mais abundantes $(185,98)$ mostrou que elas têm uma grande participação na estrutura da vegetação que está se regenerando. Após ajuste das distribuições diamétricas das sete espécies, seis apresentaram um padrão de distribuição na forma de J invertido, refletindo regeneração contínua dos indivíduos dessas espécies. Quatro dessas distribuições foram distinguidas como tipo I (ou distribuição balanceada). As características relacionadas com as distribuições de diâmetros ajustadas auxiliaram na indicação de uma atenuação da perturbação antropogênica. Verificou-se relação direta entre o arranjo espacial e a síndrome de dispersão para Trichilia clausseni e Metrodorea nigra e, dependendo das escalas analisadas, para T. catigua e T. casaretti.
\end{abstract}

Palavras-chave: comunidades, distribuição diamétrica, estatística espacial, estrutura, florestas, florística, padrão.

\section{Diameter Structure and Spatial Arrangement of the Most Abundant Species in a Seasonal Semideciduous Forest Fragment in Botucatu, Southeastern Brazil}

\begin{abstract}
We evaluated the diameter distribution and spatial arrangement of the most abundant species in one of the five forest fragments occurring on Edgardia Experimental Farm (1,200 ha) in Botucatu, Sao Paulo state, southeastern Brazil (between latitudes $22^{\circ} 47^{\prime} 30^{\prime \prime} S$ and $22^{\circ} 50^{\prime \prime} \mathrm{S}$ and longitudes $48^{\circ} 26^{\prime} 15^{\prime \prime} \mathrm{W}$ and $\left.48^{\circ} 22^{\prime} 30^{\prime \prime} \mathrm{W}\right)$. The fragment area is 56 ha large. We sampled 185 contiguous plots of $100 \mathrm{~m}^{2}(1.85 \mathrm{ha})$, accessing the floristic composition and horizontal structure of the vegetation in the center of the fragment. Among the ten species with the highest importance values, seven presented abundance values which allowed studies on diameter distribution and spatial arrangement. The sum of the importance value indexes (IVI) obtained for the most abundant species (185.98) showed that they presented significant weight in the vegetation
\end{abstract}


structure, indicating its regeneration. After adjusting the diameter distributions of the seven species, six showed an inverted J curve of their distribution pattern, reflecting continuous regeneration of plants of such species. Four of these distributions were distinguished as type I, or balanced distribution. These patterns of diameter distributions indicated attenuation of anthropogenic disturbances. There was a direct relationship between spatial arrangement and dispersal syndrome for Trichilia clausseni and Metrodorea nigra and, depending on the scales analyzed, for T. catigua and T. casaretti.

Keywords: communities, diameter distribution, spatial statistics, structure, forests, floristic, pattern.

\section{INTRODUÇÃO}

Decorrente de ações antropogênicas, as florestas estacionais semideciduais do interior paulista encontram-se fragmentadas. O desafio de conservar a biodiversidade regional em paisagens intensamente modificadas tem como principal limitante o processo de degradação de fragmentos florestais (Viana \& Pinheiro, 1998).

O presente trabalho visou estudar a vegetação natural de um dos cinco fragmentos florestais que ocorrem na Fazenda Experimental Edgardia, unidade de ensino e pesquisa administrada pela Universidade Estadual Paulista - UNESP/Campus Botucatu - São Paulo. A localização em área de proteção ambiental, o tamanho dos fragmentos e a possibilidade de ligações com outros remanescentes através de corredores ecológicos atribuem à área grande importância para a Bacia do Rio Capivara, na qual a propriedade se insere. Essa importância leva à necessidade de conservação. Para conservar a vegetação natural é preciso entender seu comportamento frente aos distúrbios naturais e antropogênicos. Procurando contribuir para esse entendimento e agregando os artigos de Silva \& Engel (1993), Fonseca \& Rodrigues (2000), Fonseca \& Fonseca (2004), referentes ao remanescente, e de Jorge et al. (2011), referente a área vizinha, analisaram-se nesta pesquisa, a estrutura diamétrica e o arranjo espacial das espécies mais abundantes que ocorrem no fragmento florestal avaliado. Algumas investigações têm auxiliado a compreender os aspectos da estrutura populacional relacionados a padrões da distribuição diamétrica e/ou de distribuição espacial de espécies arbóreas que ocorrem em fragmentos de florestas tropicais. Dentre os estudos, incluem-se os desenvolvidos por Bernasol \& Lima-Ribeiro (2010), Dalmaso et al. (2012), Silvestre et al. (2012), Watzlawick et al. (2013).
Loetsch et al. (1973) descreveram três tipos de distribuição diamétrica decrescente. $\mathrm{O}$ decréscimo do número de árvores ocorre em progressão geométrica: 1. Uniforme (tipo I); 2. Crescente (tipo II); 3. Decrescente (tipo III). Os autores reportaram que em muitas investigações relacionadas a populações de árvores tropicais as distribuições, em maior proporção, foram caracterizadas como sendo de tipo I ou II do que de tipo III. A distribuição de tipo III representa a distribuição típica para a comunidade de espécies arbóreas tropicais.

Podem ser reconhecidos dois padrões principais de dispersão de espécies arbóreas em florestas naturais, embora eles sejam parte de um continuum, sendo eles: aleatório (quando existe uma probabilidade igual de um organismo ocupar qualquer ponto no espaço, independentemente da posição de qualquer outro); e agregado (que ocorre ou quando os indivíduos tendem a ser atraídos para, ou são mais propensos a sobreviver em partes específicas do ambiente, ou a presença de um indivíduo atrai ou dá origem a um outro perto dele). O padrão espacial das árvores em uma floresta é influenciado por variáveis abióticas e bióticas. Entre as abióticas estão o relevo, a disponibilidade de luz, nutrientes e água e a caracterização do solo. Entre as principais variáveis bióticas estão a fenologia e a dispersão de sementes, os processos dependentes da densidade, como competição intraespecífica e interespecífica, herbivoria e doenças (Dalmaso et al., 2012).

A atual investigação visou responder as seguintes perguntas: 1) a comunidade de espécies arbóreas estabelecida no núcleo do remanescente estudado apresenta uma distribuição diamétrica típica decrescente (exponencial)? 2a) As espécies arbóreas mais abundantes que ocorrem no núcleo do fragmento apresentam distribuição diamétrica exponencial? 2b) Em caso afirmativo, de que tipo? 3) Os padrões verificados 
permitem inferir sobre o processo de regeneração natural que vem ocorrendo no núcleo do fragmento? 4) Existe uma relação direta entre o arranjo espacial e a síndrome de dispersão das espécies mais abundantes que ocorrem no núcleo do remanescente florestal? Para isso, o trabalho teve como objetivos: 1) Levantar a composição florística da vegetação do fragmento de floresta estacional semidecidual; 2) Caracterizar a estrutura horizontal da vegetação de tal forma a determinar as espécies mais abundantes; 3) Analisar a distribuição diamétrica e a distribuição espacial de cada uma das espécies arbóreas mais abundantes dentre as dez de maior valor de importância na área.

\section{MATERIAL E MÉTODOS}

\section{1. Área de estudo}

$\mathrm{O}$ fragmento florestal estudado, que constitui uma área de 56 ha, encontra-se na Fazenda Experimental Edgardia (área total de 1.200 ha), município de Botucatu, estado de São Paulo, e situa-se entre as coordenadas geográficas $22^{\circ} 47^{\prime} 30^{\prime \prime}$ e $22^{\circ} 50^{\prime}$ de latitude $S$ e $48^{\circ} 26^{\prime} 15^{\prime \prime}$ e $48^{\circ} 22^{\prime} 30^{\prime \prime}$ de longitude W. No local são observadas duas unidades geomórficas: as cuestas basálticas, referentes à ocorrência de rochas eruptivas básicas e seus produtos de alteração, e a depressão periférica, compreendendo a presença de arenitos e de sedimentos aluviais, principalmente a leste, abrangendo a várzea da Bacia do Rio Capivara, rebaixada 250 metros em relação às cuestas basálticas (Carvalho et al., 1991).

O clima da região tem duas estações distintas, uma chuvosa e quente (de setembro até março) e outra seca e fria (de abril até agosto). A precipitação média anual é de $1.428,4 \mathrm{~mm}$. A temperatura média anual é igual a $20,3{ }^{\circ} \mathrm{C}$, com médias mensais variando de $17,1^{\circ} \mathrm{C}$ (julho) a $23,1^{\circ} \mathrm{C}$ (fevereiro) (Cunha \& Martins, 2009). Os usos do solo principais da Fazenda Edgardia são pecuária extensiva, culturas agrícolas e pequenas áreas de plantações florestais. A vegetação natural (área total de $740 \mathrm{ha}$ ) da unidade territorial perde parcialmente as folhas na estação seca. É classificada como floresta estacional semidecidual (IBGE, 2012). Estão também presentes no local remanescentes de vegetação de transição da floresta e da savana florestada (cerradão), além de florestas ciliares (Jorge, 2009; Jorge et al., 2011). O fragmento florestal sofreu no passado significativas perturbações antropogênicas, dentre essas destaca-se a exploração seletiva de árvores com importância econômica, sendo que na última intervenção, que aconteceu entre 1975 e 1980, foram retirados indivíduos de Aspidosperma polyneuron (Apocynaceae), Cariniana estrellensis (Lecythidaceae), Enterolobium contortisiliquum (Fabaceae) e Myroxylon peruiferum (Fabaceae).

\subsection{Amostragem da vegetação natural}

No levantamento da vegetação natural do fragmento florestal foram alocadas unidades contíguas de $10 \times 10 \mathrm{~m}$, compondo um bloco de 185 unidades amostrais, perfazendo uma amostra equivalente a 1,85 hectare. O bloco constituído das unidades de amostra está localizado na região nuclear do fragmento florestal (Figura 1). Nas unidades, todos os indivíduos arbóreos que apresentaram diâmetro à altura do peito (DAP) $\geq 4,8 \mathrm{~cm}$ foram numerados e inventariados. Além da mensuração do diâmetro à altura do peito, foi feita a identificação botânica de cada indivíduo. As espécies encontradas no remanescente de vegetação natural foram classificadas de acordo com sistema de classificação botânica APG III, consultando-se a Lista de Espécies da Flora do Brasil (JBRJ, 2014).

Para a análise da distribuição espacial foram mapeados os indivíduos das espécies mais abundantes que ocorreram na área amostrada, com base na metodologia descrita por Figueiredo \& Cunha (2007), utilizando-se GPS com antena de alta sensibilidade, o que permitiu captar os sinais dos satélites mesmo sob cobertura da copa das árvores. Por questões operacionais, o mapeamento incluiu os indivíduos com DAP mínimo de $10 \mathrm{~cm}$, exceto para aquelas espécies de sub-bosque que apresentaram indivíduos com pequenas dimensões, considerando-se nesses casos um diâmetro de inclusão de $4,8 \mathrm{~cm}$.

\subsection{Análise dos dados}

No estudo sobre a estrutura horizontal da vegetação, os parâmetros quantitativos incluídos foram densidade absoluta (DA), densidade relativa (DR), dominância absoluta (DoA), dominância relativa (DoR), frequência absoluta (FA) e frequência relativa (FR), sendo também expressos na forma de índice de importância (IVI), resultante da soma dos valores relativos desses 

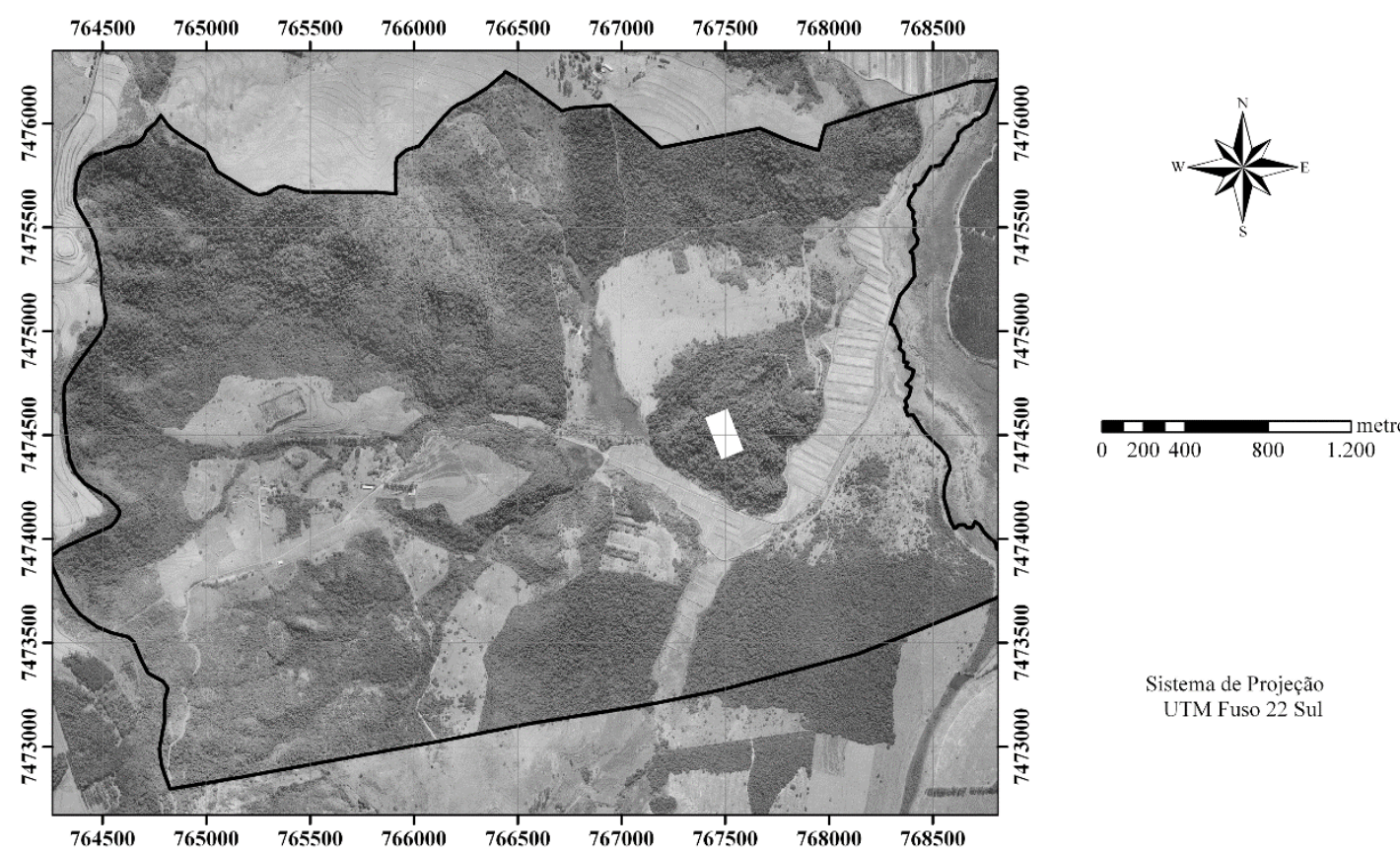

Figura 1. Área amostrada (retângulo em branco) sobre fotografia aérea que apresenta sua localização no fragmento florestal estudado.

Figure 1. Sampled area (rectangle in white) on an aerial photograph that shows its location in the fragment under study.

estimadores (Müeller-Domboi \& Ellemberg, 1974). Os cálculos dos estimadores relacionados à estrutura horizontal da amostra de 1,85 hectare foram obtidos após o desenvolvimento do módulo correspondente, utilizando-se para isso a linguagem de programação do Microsoft Access que é o VBA - Visual Basic for Applications.

Foram geradas as distribuições diamétricas do total da amostra e das espécies mais abundantes dentre as dez de maior valor de importância na área inventariada. O número de classes de diâmetro foi definido pela fórmula de Sturges (Machado \& Figueiredo, 2003).

As frequências observadas por classe de diâmetro foram computadas a partir do desenvolvimento do módulo correspondente, que foi programado no VBA. Procurando-se descrever de maneira mais detalhada o comportamento das distribuições de diâmetro que mostraram um padrão em J invertido, ajustaram-se três modelos exponenciais (Tabela 1). O melhor ajuste permitiu indicar se o decréscimo do número de árvores relacionadas a uma distribuição específica se aproximou de uma progressão geométrica uniforme
- tipo I, crescente - tipo II ou decrescente - tipo III (Loetsch et al., 1973).

Tendo como base os modelos linearizados (Tabela 1), foram utilizadas as equações lineares como funções de autoiniciação para o ajuste das equações não lineares no ambiente do aplicativo estatístico R (Ritz \& Streibig, 2008), sendo os valores iniciais dos coeficientes a, b assumidos respectivamente por $\mathrm{e}^{\mathrm{b} 0}, \mathrm{~b}_{1}$.

Quando as frequências observadas de uma determinada espécie apontaram para um padrão de distribuição de diâmetros unimodal, empregaram-se três modelos (Tabela 2) adaptados para seu uso na presente pesquisa. Os modelos linearizados foram ajustados. Selecionou-se a melhor equação em função do coeficiente de determinação $\left(R^{2}\right)$, erro padrão de estimativa (syx) e significância dos coeficientes. Após derivar o antilogaritmo da equação escolhida, descreveu-se a distribuição estimada.

Dentre outras estatísticas, a qualidade do ajuste das equações utilizadas para descrever as distribuições diamétricas, tanto para as que apresentaram um padrão 
Tabela 1. Modelos ajustados para o padrão de distribuição de diâmetros em J invertido.

Table 1. Models adjusted for inverted J curve of diameter distribution.

\begin{tabular}{cccc} 
Tipo & Progressão geométrica & Modelo exponencial & Modelo linearizado \\
I & Uniforme & $1 . N_{i}=a e^{b d_{i}}$ & $1 . \ln N_{i}=b_{0}+b_{1} d_{i}$ \\
II & Crescente & $2 . N_{i}=a e^{b d_{i}^{2}}$ & $2 \cdot \ln N_{i}=b_{0}+b_{1} d_{i}^{2}$ \\
III & Decrescente & $3 . N_{i}=a e^{b d_{i}^{1 / 2}}$ & $3 \cdot \ln N_{i}=b_{0}+b_{1} d_{i}^{1 / 2}$ \\
\hline
\end{tabular}

$\mathrm{N}_{\mathrm{i}}=$ número de indivíduos na classe $\mathrm{i} ; \mathrm{d}_{\mathrm{i}}=$ centro da classe de diâmetros $\mathrm{i} ; \mathrm{a}, \mathrm{b}=$ coeficientes dos modelos exponenciais; $\mathrm{b}_{0}, \mathrm{~b}_{1}=$ coeficientes dos modelos linearizados.

Tabela 2. Modelos ajustados para o padrão de distribuição de diâmetros unimodal.

Table 2. Models adjusted for unimodal diameter distribution.

\begin{tabular}{cc} 
Modelo & Modelo linearizado \\
1. $N_{i}=a d_{i}^{b} e^{c d_{i}}$ & 1. $\ln N_{i}=b_{0}+b_{1} \ln d_{i}+b_{2} d_{i}$ \\
2. $N_{i}=a d_{i}^{b} e^{c d_{i}^{2}}$ & 2. $\ln N_{i}=b_{0}+b_{1} \ln d_{i}+b_{2} d_{i}^{2}$ \\
3. $N_{i}=a d_{i}^{b} e^{c d_{i}^{1 / 2}}$ & 3. $\ln N_{i}=b_{0}+b_{1} \ln d_{i}+b_{2} d_{i}^{1 / 2}$ \\
\hline
\end{tabular}

$\mathrm{N}_{\mathrm{i}}=$ número de indivíduos na classe $\mathrm{i} ; \mathrm{d}_{\mathrm{i}}=$ centro da classe de diâmetros i; a, b, c = coeficientes dos modelos não lineares; $b_{0}$, $\mathrm{b}_{1}, \mathrm{~b}_{2}=$ coeficientes dos modelos linearizados.

em J invertido como unimodal, foi medida pelo índice de ajustamento (IA), calculado pela Fórmula 1:

$I A=100\left[1-\frac{\sum_{i=1}^{n}\left(y_{i}-\hat{y}_{i}\right)^{2}}{\sum_{i=1}^{n}\left(y_{i}-\bar{y}_{i}\right)^{2}}\right]$

em que:

$y_{i}=$ frequência absoluta observada na classe i;

$\hat{y}_{i}=$ frequência absoluta esperada na classe i;

$\bar{y}_{i}=$ frequência média nas $\mathrm{n}$ classes.

Os mapas de pontos contendo a posição de cada indivíduo das espécies em que se avaliou a distribuição espacial foram elaborados no ambiente do aplicativo ArcInfo v.10, com base nos arquivos do GPS. Em um segundo momento, os dados foram transferidos para o ambiente do aplicativo R, utilizando-se o pacote spatstat (Baddeley, 2010).

A função K de Ripley computa as distâncias entre todas as árvores em um mapa (Cruz-Rot, 2006). De modo simplificado, o procedimento consiste em gerar um círculo de raio r centrado em cada árvore. $\mathrm{O}$ número de vizinhos dentro de cada círculo é contado. Variando o raio r é possível detectar o padrão espacial em diferentes escalas. Para determinar o padrão de distribuição dos indivíduos das espécies mais abundantes empregou-se, em concordância com Cruz-Rot (2006), Dalmaso (2012) e Dalmaso et al. (2012), a função L, que é uma transformada da função K univariada $\left\{\mathrm{L}(\mathrm{r})=[\mathrm{K}(\mathrm{r}) / \pi]^{1 / 2}\right\}$. Testes de significância para as medidas espaciais são geralmente construídos utilizando-se simulações de Monte Carlo (Diggle, 2003). Para a função univariada, a hipótese nula é a da completa aleatoriedade espacial (testar se $\mathrm{L}(\mathrm{r})-\mathrm{r}=0$ a cada distância $\mathrm{r}$ ). A hipótese foi testada no spatstat por 500 simulações Monte Carlo para $\alpha=0,01$. Criaram-se os envelopes de confiança. Assim, para os valores observados que estavam dentro de tais envelopes considerou-se o padrão espacial como aleatório. Para valores positivos encontrados fora dos envelopes de confiança rejeitou-se a hipótese nula e considerou-se o padrão espacial como agregado.

\section{RESULTADOS E DISCUSSÃO}

O levantamento fitossociológico quantificou 2.121 indivíduos de 29 famílias e 75 espécies (Tabela 3). Dentre as dez espécies de maior valor de importância (Figura 2), sete apresentaram abundância que permitiu realizar, em relação a elas, os estudos sobre distribuição diamétrica e distribuição espacial. Os somatórios dos índices de valores de importância (IVI) obtidos para as sete espécies mais abundantes, para as outras três dentre as dez com maiores IVIs e para as demais 65 espécies encontradas no levantamento foram respectivamente $185,98,29,03$ e 84,99 .

Além da distribuição de diâmetros da comunidade de espécies arbóreas inventariadas (Tabela 4; Figura 3a), ajustaram-se as distribuições das espécies mais abundantes dentre as dez de maior valor de importância: Trichilia claussenii (Tabela 4; Figura 3b), Aspidosperma polyneuron (Tabela 4; Figura 3c), Gallesia integrifolia (Tabela 4; Figura 3d), Holocalyx balansae (Tabela 4; 
Tabela 3. Composição florística do centro do fragmento florestal.

Table 3. Floristic composition of the forest fragment.

\begin{tabular}{|c|c|c|}
\hline Família & Nome científico & Nome vulgar \\
\hline Anacardiaceae & Astronium graveolens Jacq. & Guaritá \\
\hline Annonaceae & Annona sylvatica A. St.-Hil. & Araticum-da-mata \\
\hline \multirow[t]{5}{*}{ Apocynaceae } & Actinostemon concepcionis (Chodat e Hass 1.) Hochr. & Guatambu-vermelho \\
\hline & Aspidosperma cylindrocarpon Müll. Arg. & Peroba-poca \\
\hline & Aspidosperma polyneuron Müll. Arg. & Peroba-rosa \\
\hline & Aspidosperma ramiflorum Müll. Arg. & Guatambu-amarelo \\
\hline & Aspidosperma subincanum Mart. & Guatambu-vermelho \\
\hline \multirow[t]{2}{*}{ Arecaceae } & Syagrus oleracea (Mart.) Becc. & Palmito amargo \\
\hline & Syagrus romanzoffiana (Cham.) Glassman & Jerivá \\
\hline \multirow[t]{3}{*}{ Boraginaceae } & Cordia americana (L.) Gottschling e J. S. Mill. & guajuvira \\
\hline & Cordia ecalyculata Vell. & Café-de-bugre \\
\hline & Cordia trichotoma (Vell.) Arráb. ex Steud. & Louro-pardo \\
\hline Cactaceae & Cereus hildmannianus K.Schum. & Mandacaru \\
\hline \multirow[t]{2}{*}{ Cannabaceae } & Celtis fluminensis Carauta & Grão-de-galo \\
\hline & Trema micranta (L.) Blume & Grandiúva \\
\hline Caricaceae & Jacaratia spinosa (Aubl.) A. DC. & Mamãozinho-do-mato \\
\hline Celastraceae & Maytenus aquifolia Mart. & Espinheira-santa \\
\hline Euphorbiaceae & Actinostemon klotzschii (Didr.) Pax & Falsa-guajuvira \\
\hline \multirow[t]{3}{*}{ Fabaceae - Caesalpinioideae } & Cassia ferrugínea (Schrad.) Schrad. ex DC. & Chuva-de-ouro \\
\hline & Copaifera langsdorffii Desf. & Copaíba \\
\hline & Holocalyx balansae Micheli & Alecrim-de-campinas \\
\hline \multirow[t]{9}{*}{ Fabaceae - Faboideae } & Centrolobium tomentosum Guill. ex Benth. & Araribá \\
\hline & Dalbergia frutescens (Vell.) Britton & Canudo-de-pito \\
\hline & Lonchocarpus muehlbergianus Hassl. & Embira-de-sapo \\
\hline & Machaerium brasiliense Vogel & Jacarandá \\
\hline & Machaerium nyctitans (Vell.) Benth. & Jacanradá-bico-de-pato \\
\hline & Machaerium scleroxylon Tul. & Caviúna \\
\hline & Machaerium stipitatum Vogel & Sapuva \\
\hline & Myroxylon peruiferum L.f. & Cabreúva-vermelha \\
\hline & Pterocarpus rohrii Vahl & Aldrago \\
\hline \multirow[t]{5}{*}{ Fabaceae - Mimosoideae } & Anadenanthera colubrina (Vell.) Brenan & Angico-branco \\
\hline & Calliandra foliolosa Benth. & Topete-de-cardeal \\
\hline & Enterolobium contortisiliquum (Vell.) Morong & Tamboril \\
\hline & Parapiptadenia rígida (Benth.) Brenan & Angico-vermelho \\
\hline & Senegalia polyphylla (DC.) Britton e Rose & Monjoleiro \\
\hline Lecythidaceae & Cariniana estrellensis (Raddi) Kuntze & Jequitibá-branco \\
\hline \multirow[t]{3}{*}{ Malvaceae } & Ceiba speciosa (A. St.-Hil.) Ravenna & Paineira \\
\hline & Guazuma ulmifolia Lam. & Mutamba \\
\hline & Bastardiopsis densiflora (Hook. e Arn.) Hassl. & Pau-jangada-brava \\
\hline \multirow[t]{4}{*}{ Meliaceae } & Trichilia casaretti C. DC. & Baga-de-morcego \\
\hline & Trichilia catigua A. Juss. & Catiguá, catuaba \\
\hline & Trichilia claussenii C. DC. & Catiguá vermelho \\
\hline & Trichilia elegans A. Juss. & Pau-ervilha \\
\hline \multirow[t]{8}{*}{ Myrtaceae } & Calycorectes acutatus (Miq.) Toledo & Araçá-da-serra \\
\hline & Calyptranthes concinna DC. & Guamirim-facho \\
\hline & Campomanesia guazumifolia (Cambess.) O. Berg & Sete-capotes \\
\hline & Campomanesia xanthocarpa (Mart.) O. Berg & Gabiroba árvore \\
\hline & Eugenia blasthanta (O. Berg) D. Legrand & Grumixama miúda \\
\hline & Eugenia glazioviana Kiaersk & Guamirim \\
\hline & Eugenia uniflora L. & Pitanga \\
\hline & Eugenia myrcianthes Nied. & Laranjinha-do-mato \\
\hline
\end{tabular}


Tabela 3. Continuação...

Table 3. Continued...

\begin{tabular}{|c|c|c|}
\hline Família & Nome científico & Nome vulgar \\
\hline & Myrcianthes pungens (O. Berg) D.Legrand & Guabirobaçu \\
\hline & Myrciaria floribunda (H.West ex Willd.) O. Berg & Murtinha do campo \\
\hline & Psidium sartorianum (O. Berg) Nied. & Cambuí \\
\hline Nyctaginaceae & Guapira opposita (Vell.) Reitz & Maria-mole \\
\hline Phyllanthaceae & Savia dictyocarpa Müll. Arg. & Guaraiúva \\
\hline Phytolaccaceae & Gallesia integrifólia (Spreng.) Harms & Pau-d'alho \\
\hline Picramniaceae & Picramnia ramiflora Planch. & Picrâmnia, camboitá \\
\hline \multirow[t]{2}{*}{ Rhamnaceae } & Colubrina glandulosa Perkins & Saguaraji vermelho \\
\hline & Rhamnidium elaeocarpum Reissek & Saguaraji amarelo \\
\hline Rosaceae & Prunus myrtifolia (L.) Urb. & Pessegueiro-bravo \\
\hline Rubiaceae & Ixora gardneriana Benth. & Ixora \\
\hline \multirow[t]{4}{*}{ Rutaceae } & Balfourodendron riedelianum (Engl.) Engl. & Pau-marfim \\
\hline & Esenbeckia febrífuga (A. St.-Hil.) A. Juss. ex Mart. & Canela-de-cutia falsa \\
\hline & Esenbeckia grandiflora Mart. & Canela-de-cutia verdadeira \\
\hline & Metrodorea nigra A. St.-Hil. & Carrapateira \\
\hline \multirow[t]{2}{*}{ Salicaceae } & Casearia gossypiosperma Briq. & Pau-de-espeto \\
\hline & Casearia sylvestris Sw. & Guaçatonga, largarteira \\
\hline \multirow[t]{2}{*}{ Sapindaceae } & Allophylus edulis (A. St.-Hil. et al.) Hieron. ex Niederl. & Chal-chal \\
\hline & Diatenopteryx sorbifolia Radlk. & Maria-preta \\
\hline Sapotaceae & Chrysophyllum gonocarpum (Mart. e Eichler ex Miq.) Engl. & Guatambu-de-leite \\
\hline \multirow[t]{2}{*}{ Solanaceae } & Solanum argenteum Dunal & Folha-de-prata \\
\hline & Solanum pseudoquina A. St.-Hil. & Joá-árvore \\
\hline Urticaceae & Urera baccifera (L.) Gaudich. ex Wedd. & Urtigão \\
\hline Verbenaceae & Aloysia virgata (Ruiz e Pav.) Juss. & Lixeira \\
\hline
\end{tabular}

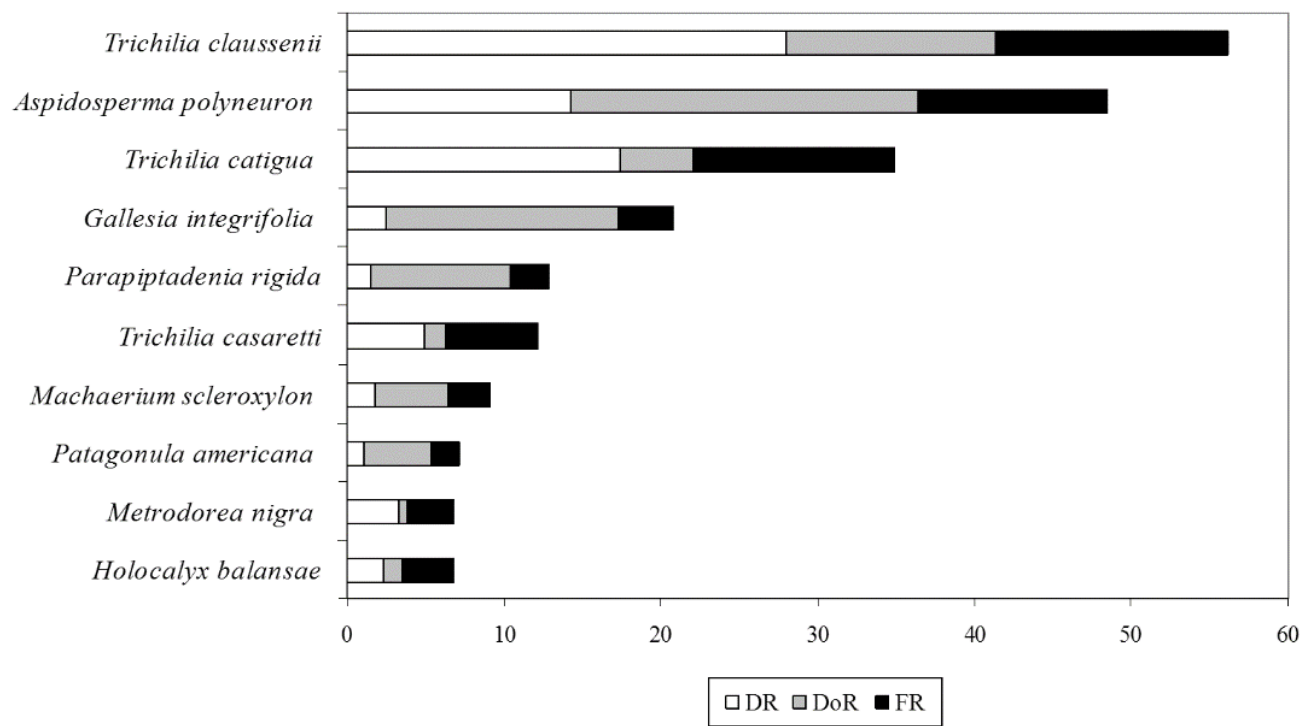

Figura 2. Dez espécies de maior valor de índice de importância; o valor da soma dos IVIs das outras espécies é igual a 84,99 .

Figure 2. Ten species with the highest importance index values; the value of the sum of the IVIs of the other species is 84.99 . 
Tabela 4. Estatísticas relacionadas às equações ajustadas para o padrão de distribuição de diâmetros em J invertido. Table 4. Statistics related to the equations adjusted for inverse-J-shaped diameter distribution.

\begin{tabular}{|c|c|c|c|c|c|c|}
\hline Distribuição & Modelo & a & b & syx & $\begin{array}{c}\text { n. de } \\
\text { iterações }\end{array}$ & IA (\%) \\
\hline \multirow[t]{3}{*}{ Comunidade } & 1 & $4423,2444238^{\star * *}$ & $-0,1329894^{\star * \star}$ & 25,880 & 6 & 99,67 \\
\hline & 2 & $2055,4700000^{\star * *}$ & $-0,0050529^{\star * \star}$ & 47,560 & 8 & 98,88 \\
\hline & 3 & $25611,8932640^{* * *}$ & $-0,9891210^{* * *}$ & 9,441 & 4 & 99,96 \\
\hline \multirow[t]{3}{*}{ Trichilia claussenii } & 1 & $632,7941226^{* * *}$ & $-0,1238066^{\star * *}$ & 5,809 & 6 & 99,65 \\
\hline & 2 & $339,4755625^{\star * *}$ & $-0,0051207^{\star * *}$ & 6,596 & 4 & 99,55 \\
\hline & 3 & $2645,7229505^{\star * *}$ & $-0,8702356^{* * *}$ & 12,890 & 11 & 98,27 \\
\hline \multirow[t]{3}{*}{ Aspidosperma polyneuron } & 1 & $159,0425452^{* * *}$ & $-0,0576243^{\star \star *}$ & 3,987 & 2 & 98,82 \\
\hline & 2 & $105,1417982^{* * *}$ & $-0,0015382^{* * *}$ & 7,863 & 10 & 95,40 \\
\hline & 3 & $400,6194938^{* * *}$ & $-0,4843399^{* * *}$ & 5,853 & 6 & 97,45 \\
\hline \multirow[t]{3}{*}{ Gallesia integrifolia } & 1 & $22,2999353^{\star * *}$ & $-0,0212393^{\star \star}$ & 1,816 & 4 & 91,04 \\
\hline & 2 & $16,4727252^{\star * *}$ & $-0,0002591^{\star *}$ & 2,187 & 5 & 87,00 \\
\hline & 3 & $40,2317634^{\star \star}$ & $-0,2388776^{\star \star}$ & 2,003 & 6 & 89,10 \\
\hline \multirow[t]{3}{*}{ Holocalyx balansae } & 1 & $104,0755984^{*}$ & $-0,1552170^{\star \star}$ & 2,677 & 7 & 96,37 \\
\hline & 2 & $48,5143088^{* *}$ & $-0,0071619^{\star}$ & 3,517 & 14 & 93,83 \\
\hline & 3 & 622,3602290 & $-1,0758800^{\star *}$ & 2,287 & 4 & 97,20 \\
\hline \multirow[t]{3}{*}{ Metrodorea nigra } & 1 & $132,7289296^{*}$ & $-0,2327810^{\star}$ & 4,269 & 8 & 93,30 \\
\hline & 2 & $55,6382739^{* *}$ & $-0,0138865^{\star *}$ & 3,173 & 8 & 96,30 \\
\hline & 3 & 881,2126710 & $-1,3516880^{*}$ & 4,927 & 10 & 91,07 \\
\hline \multirow[t]{3}{*}{ Trichilia catigua } & 1 & $946,7068707^{* * *}$ & $-0,2104805^{\star * *}$ & 6,391 & 4 & 99,61 \\
\hline & 2 & $378,3017510^{* * *}$ & $-0,0108511^{\star * *}$ & 12,220 & 7 & 98,63 \\
\hline & 3 & $7401,8643620^{\star *}$ & $-1,3411710^{* * *}$ & 6,365 & 9 & 99,60 \\
\hline
\end{tabular}

${ }^{* *} \mathrm{p} \leq 0,001 \cdot{ }^{* *} \mathrm{p} \leq 0,01 \cdot{ }^{*} \mathrm{p} \leq 0,05 \cdot \mathrm{p} \leq 0,1$.

Figura 3e), Metrodorea nigra (Tabela 4; Figura 3f), Trichilia catigua (Tabela 4; Figura 3g) e Trichilia casaretti (Tabela 5; Figura 3h).

As distribuições de diâmetro da comunidade de espécies arbóreas e de Trichilia claussenii, Aspidosperma polyneuron, Gallesia integrifolia, Holocalyx balansae, Metrodorea nigra e Trichilia catigua apresentaram um padrão na forma de J invertido, enquanto a população de $T$. casaretti mostrou uma distribuição unimodal com assimetria positiva.

Para determinar o tipo de distribuição decrescente entre aquelas que apresentaram o padrão em J invertido, observou-se o ajuste dos três modelos exponenciais testados (Tabela 4). Para a escolha da equação mais adequada para descrever cada distribuição foi analisada a que exibiu o menor erro padrão de estimativa (syx), coeficientes com nível de significância menor ou igual a $5 \%$ e índice de ajustamento (IA) maior ou igual a 90\%. Sendo assim, para a comunidade de espécies arbóreas, selecionou-se a equação baseada no modelo 3 , que caracteriza uma distribuição tipo III, em que o número de indivíduos decresce nas classes de diâmetro, aproximando-se de uma progressão geométrica decrescente, distribuição típica para o conjunto das espécies de áreas de florestas tropicais (Loetsch et al., 1973). Para T. claussenii, A. polyneuron, G. integrifolia e H. balansae foram escolhidas as equações baseadas no modelo 1, que caracteriza uma distribuição tipo I (distribuição diamétrica balanceada), em que o número de indivíduos decresce nas classes de diâmetro, acercando-se de uma progressão geométrica uniforme. A equação baseada no modelo 2 , que caracteriza uma distribuição tipo II, foi selecionada para M. nigra. Nesse tipo de distribuição, o número de indivíduos decresce nas classes de diâmetro, aproximando-se de uma progressão geométrica crescente. A distribuição de diâmetros de T. catigua exibiu o mesmo padrão (J invertido - tipo III) encontrado para a comunidade de espécies arbóreas.

Para os indivíduos T. claussenii (Figuras 4a e 5a), o padrão de distribuição espacial foi aleatório entre 0 e 1 metro e agregado na escala de 1 até 30 metros. A. polyneuron (Figuras $4 \mathrm{~b}$ e $5 \mathrm{~b}$ ) apresentou padrão aleatório entre 0 e 3 metros e agregado na escala de 
3 a 30 metros. G. integrifolia (Figuras 4c e 5c) mostrou um padrão aleatório entre 0 e 1 metro e agregado na escala de 1 a 30 metros. O padrão de distribuição espacial de H. balansae (Figuras 4d e 5d) foi aleatório, em função dos valores de L(r) - r observados em todas as escalas estarem dentro do envelope de confiança. Verificou-se para M. nigra (Figuras 4e e 5e) um padrão aleatório entre 0 e 1 metro e agregado na escala de 1 a 30 metros. T. catigua (Figuras $4 \mathrm{f}$ e $5 \mathrm{f}$ ) apresentou um padrão aleatório entre 0 e 4 metros e agregado na
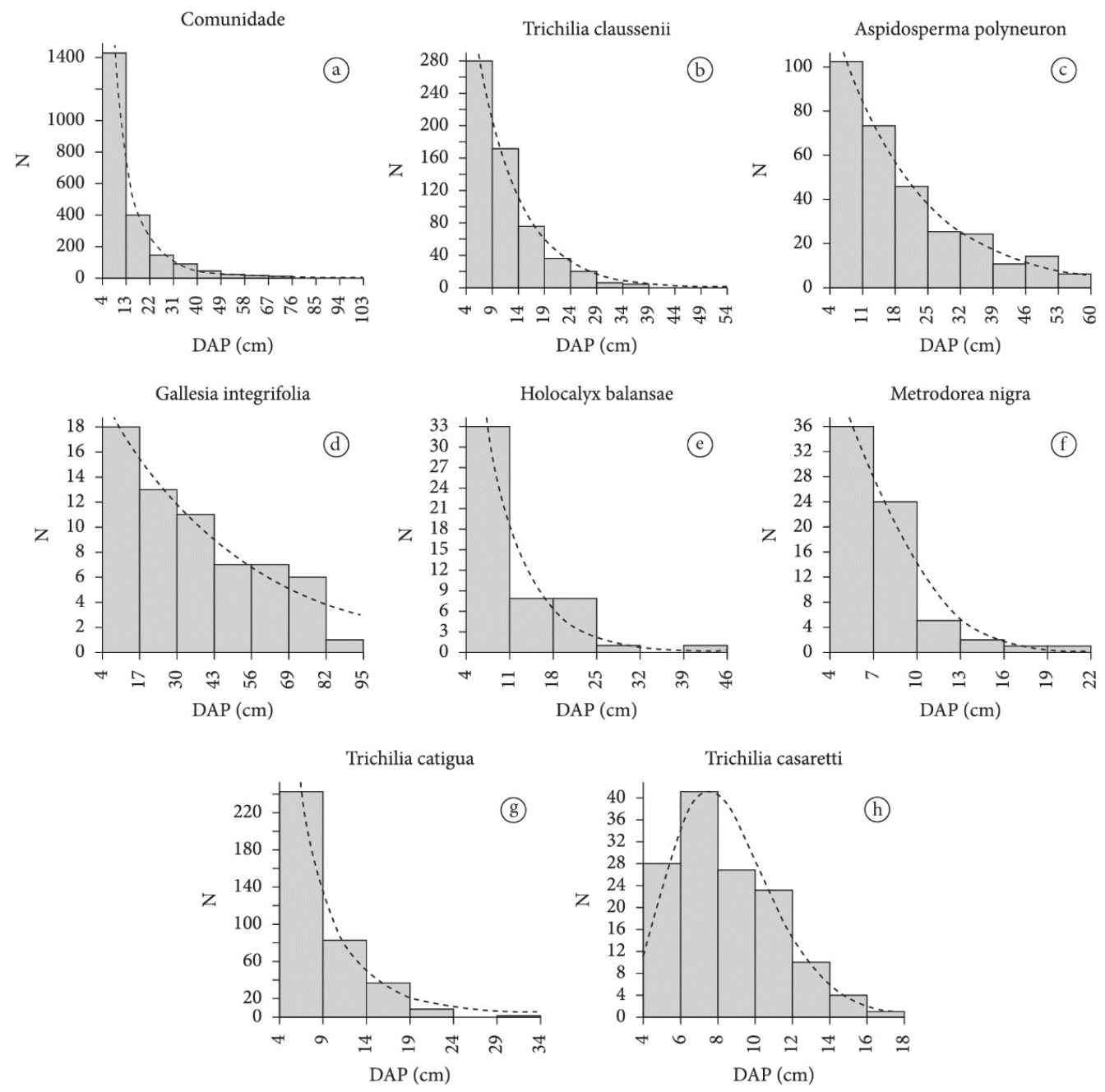

Figura 3. Distribuição de diâmetros: a) comunidade de espécies arbóreas; b) Trichilia claussenii; c) Aspidosperma polyneuron; d) Gallesia integrifolia; e) Holocalyx balansae; f) Metrodorea nigra; g) Trichilia catigua; h) Trichilia casaretti.

Figure 3. Diameter distribution of: a) community of tree species; b) Trichilia claussenii; c) Aspidosperma polyneuron; d) Gallesia integrifolia; e) Holocalyx balansae; f) Metrodorea nigra; g) Trichilia catigua; and h) Trichilia casaretti.

Tabela 5. Estatísticas relacionadas às equações ajustadas para o padrão de distribuição de diâmetros unimodal. Table 5. Statistics related to the equations adjusted for unimodal diameter distribution.

\begin{tabular}{cccccccc} 
Distribuição & Modelo & $\mathbf{b}_{\mathbf{0}}$ & $\mathbf{b}_{\mathbf{1}}$ & $\mathbf{b}_{\mathbf{2}}$ & $\mathbf{s y x}$ & $\mathbf{R}_{\text {ajust }}$ & IA (\%) \\
Trichilia casaretti & 1 & $-4,437500^{*}$ & $8,03550^{* *}$ & $-1,071200^{* * *}$ & 0,1856 & 0,9807 & 92,30 \\
& 2 & $-0,973227$ & $3,00101^{* *}$ & $-0,025954^{* * *}$ & 0,1729 & 0,9833 & 90,24 \\
& 3 & $3,700000^{* *}$ & $18,17270^{* *}$ & $-13,344000^{* * *}$ & 0,2081 & 0,9757 & 92,55 \\
\hline
\end{tabular}

${ }^{* * *} \mathrm{p} \leq 0,001 .{ }^{* *} \mathrm{p} \leq 0,01 \cdot{ }^{*} \mathrm{p} \leq 0,05$. 

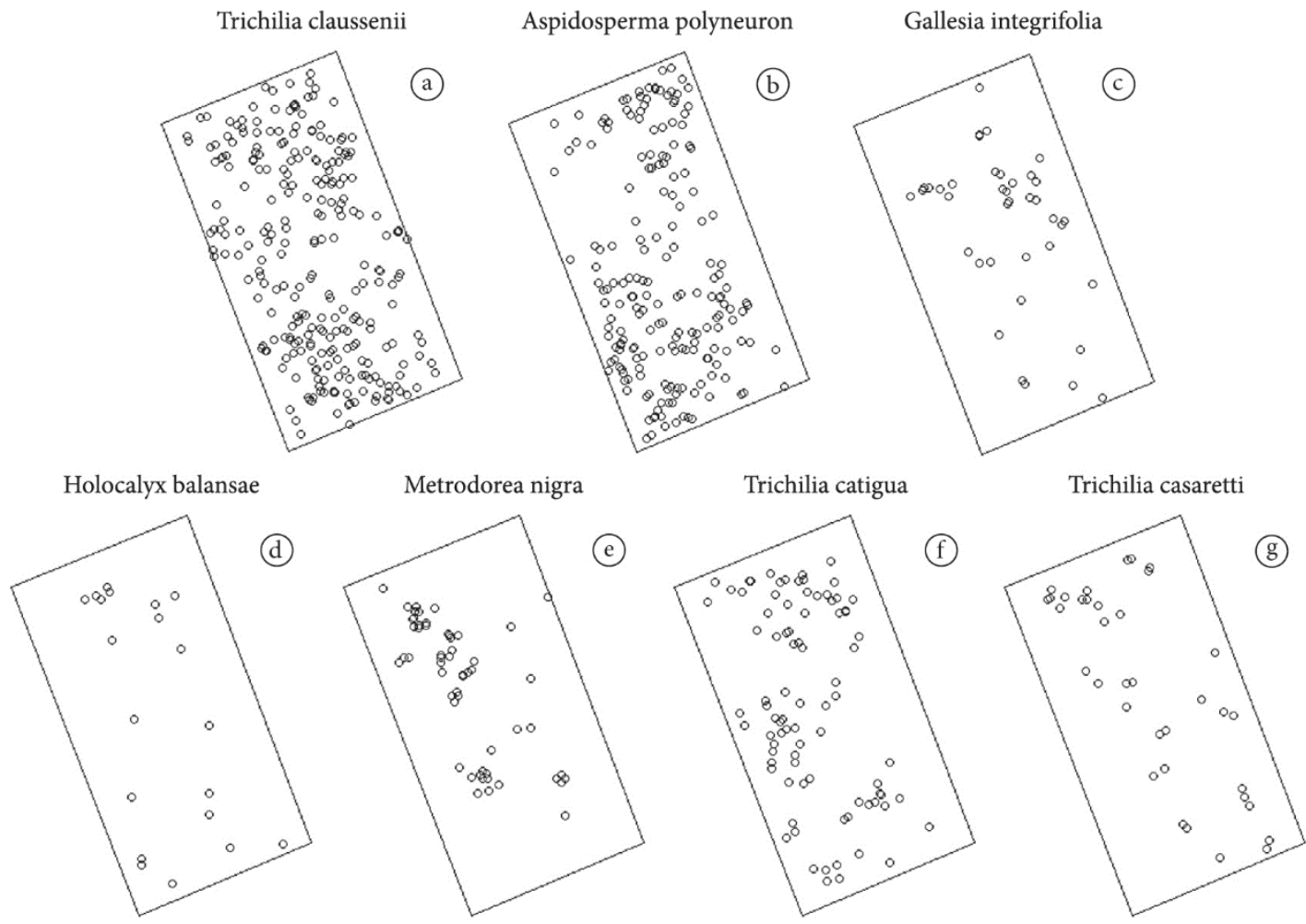

Figura 4. Distribuição espacial dos indivíduos: a) Trichilia claussenii; b) Aspidosperma polyneuron; c) Gallesia integrifolia; d) Holocalyx balansae; e) Metrodorea nigra; f) Trichilia catigua; g) Trichilia casaretti.

Figure 4. Spatial distribution of the individuals of: a) Trichilia claussenii; b) Aspidosperma polyneuron; c) Gallesia integrifolia; d) Holocalyx balansae; e) Metrodorea nigra; f) Trichilia catigua; and g) Trichilia casaretti.

escala de 4 a 30 metros. T. casaretti (Figuras $4 \mathrm{~g}$ e $5 \mathrm{~g}$ ) exibiu um padrão de distribuição dos indivíduos aleatório nas escalas de 0 a 5 metros e de 18 a 30 metros, bem como um padrão agregado entre 5 e 18 metros.

Em escalas em que os indivíduos estão muito próximos uns dos outros, a utilização da função L (transformada da função K univariada de Ripley) indicou padrão aleatório para todas as espécies estudadas.

A dispersão de sementes produzidas pela planta mãe é o processo inicial que gera a distribuição espacial de uma determinada espécie. Para uma espécie em que a dispersão ocorre pelo vento (anemocoria), seria esperada uma distribuição dos indivíduos ao acaso. Em relação à autocoria, a expectativa está na deposição das sementes/frutos em determinados microssítios, mais precisamente ao redor da planta mãe, estabelecendo-se uma distribuição agregada. Tal padrão também seria plausível para espécies dispersas por animais (zoocoria), visto que muitas vezes há a deposição de sementes em certos locais, como em poleiros de alimentação. Harms et al. (2000) e Jordano \& Godoy (2002) observaram padrões de dispersão por animais com acúmulo de sementes em determinados pontos, enquanto grande parte da área não era atingida pela disseminação, contrastando com os padrões geralmente aleatórios de espécies anemocóricas. Essas possíveis associações diretas entre padrão de distribuição espacial encontrado e síndrome de dispersão não foram observadas para todas as sete espécies mais abundantes na área de estudo (Tabela 6), o que indica que no processo para A. polyneuron, G. integrifolia e H. balansae, além da dispersão de sementes, entraram outros aspectos relacionados a características ecofisiológicas das espécies (fenologia, competição intraespecífica e interespecífica) e às condições ambientais (disponibilidade de luz, umidade, nutrientes no solo) nos microssítios em que as sementes das árvores foram depositadas. A agregação encontrada para indivíduos T. clausseni, T. catigua (escalas específicas) e T. casaretti (escalas específicas) 
está relacionada à dispersão por aves (Gondim, 2001), sendo associada a hábitos alimentares ligados a abrigos ou poleiros naturais. Em relação a $M$. nigra verificou-se relação direta entre autocoria e a distribuição agregada da espécie.
Quanto a sua ocorrência nos fragmentos de vegetação natural da Fazenda Experimental Edgardia, M. nigra foi caracterizada como tolerante de sub-bosque. Jorge et al. (2011), ao estudarem um segundo fragmento da unidade experimental, verificaram que a espécie é indicadora
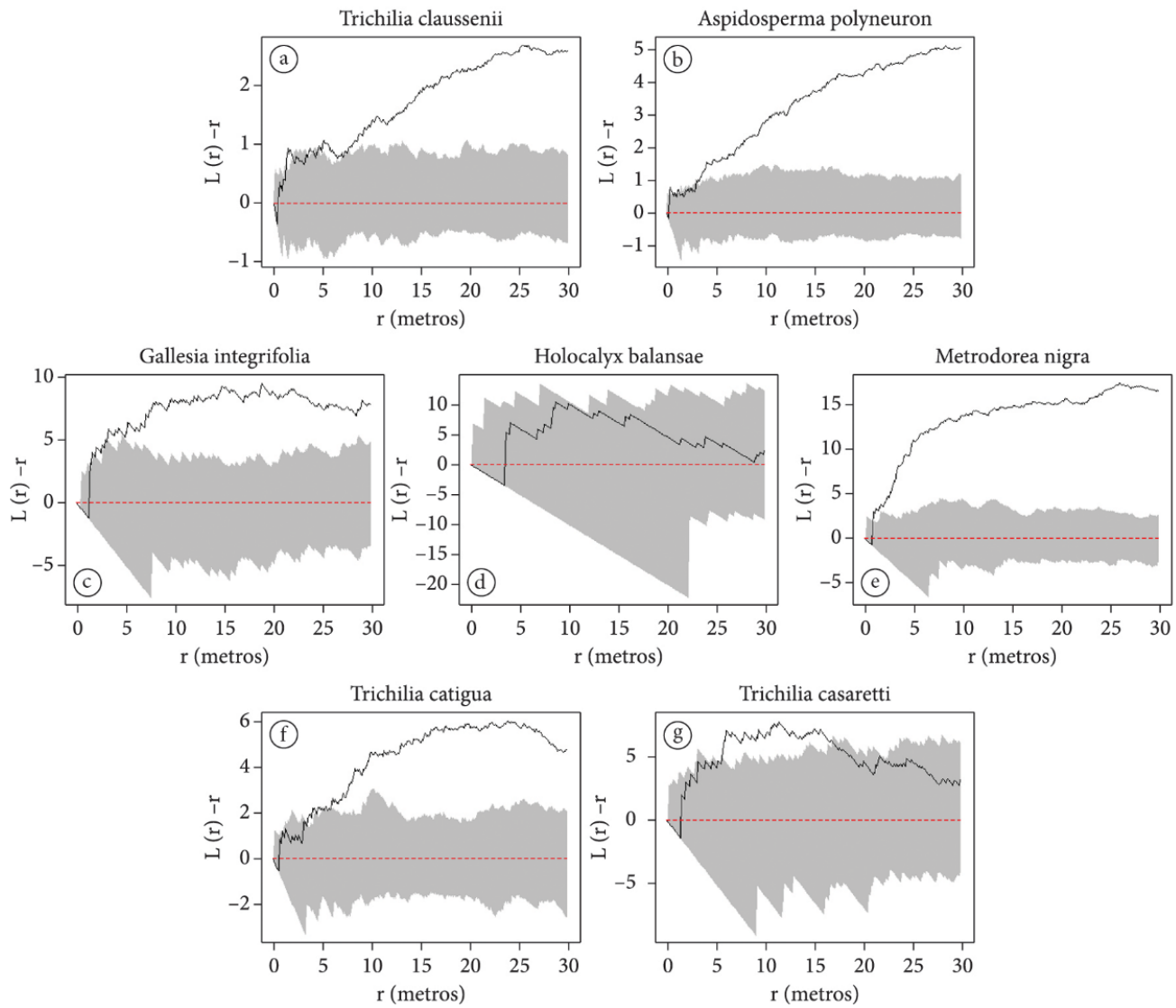

Figura 5. Função L e envelope de confiança para: a) Trichilia claussenii; b) Aspidosperma polyneuron; c) Gallesia integrifolia; d) Holocalyx balansae; e) Metrodorea nigra; f) Trichilia catigua; g) Trichilia casaretti.

Figure 5. L-function and confidence envelope for: a) Trichilia claussenii; b) Aspidosperma polyneuron; c) Gallesia integrifolia; d) Holocalyx balansae; e) Metrodorea nigra; f) Trichilia catigua; and g) Trichilia casaretti.

Tabela 6. Padrão de distribuição espacial (DE), síndrome de dispersão (SD) e grupo ecológico (GE) das espécies mais abundantes do fragmento florestal.

Table 6. Spatial distribution pattern (DE), dispersion syndrome (SD) and ecological group (GE) of the most abundant species of the forest fragment.

\begin{tabular}{lccl}
\multicolumn{1}{c}{ Espécie } & DE & SD & GE \\
Trichilia claussenii & Agregado & Zoocórica & Secundária tardia \\
Aspidosperma polyneuron & Agregado & Anemocórica & Secundária tardia \\
Gallesia integrifolia & Agregado & Anemocórica & Secundária tardia \\
Holocalyx balansae & Aleatório & Zoocórica & Secundária tardia \\
Metrodorea nigra & Agregado & Autocórica & Secundária tardia \\
Trichilia catigua & Aleatório/Agregado & Zoocórica & Secundária tardia \\
Trichilia casaretti & Aleatório/Agregado & Zoocórica & Secundária tardia \\
\hline
\end{tabular}


de áreas menos perturbadas. Os resultados obtidos no presente trabalho mostraram que a espécie tem um padrão de distribuição espacial agregado. Sendo assim, a localização dos indivíduos de $M$. nigra pode ser usada para identificar os locais menos perturbados dentro do mosaico sucessional.

\section{CONCLUSÕES}

Com base nos resultados do presente trabalho, concluiu-se que:

1) O somatório dos índices de valores de importâncias das sete espécies mais abundantes indicou que elas têm uma grande participação na estrutura da vegetação, que está regenerando no núcleo do fragmento florestal em estudo.

2) No ajuste das distribuições diamétricas das sete espécies mais abundantes dentre as dez de maior valor de importância, seis apresentaram um padrão de distribuição na forma de J invertido, refletindo regeneração contínua dos indivíduos dessas espécies. Quatro dessas distribuições foram distinguidas como tipo I ou de distribuição balanceada. As características relacionadas com as distribuições de diâmetros auxiliaram na indicação de uma atenuação da perturbação antropogênica.

3) Verificou-se relação direta entre o arranjo espacial e a síndrome de dispersão para Trichilia clausseni e Metrodorea nigra e, dependendo das escalas analisadas, para T. catigua e T. casaretti.

\section{STATUS DA SUBMISSÃO}

Recebido: 14 jan., 2013

Aceito: 21 mar., 2015

\section{AUTOR(ES) PARA CORRESPONDÊNCIA}

\section{Luiz Alberto Blanco Jorge}

Faculdade de Ciências Agronômicas, Universidade Estadual Paulista - UNESP, CEP 18610-307, Botucatu, SP, Brasil e-mail: blanco@fca.unesp.br

\section{REFERÊNCIAS}

Baddeley A. Analysing spatial point patterns in R. Perth: CSIRO:University of Western Australia; 2010. Workshop Notes, Version 4.1.

Bernasol WP, Lima-Ribeiro MS. Estrutura espacial e diamétrica de espécies arbóreas e seus condicionantes em um fragmento de cerrado sentido estrito no sudoeste goiano. Hoehnea 2010; 37(2): 181-198. http://dx.doi. org/10.1590/S2236-89062010000200001.

Carvalho WA, Panoso LA, Moraes MH. Levantamento semidetalhado dos solos da fazenda experimental Edgardia - município de Botucatu. Botucatu: Faculdade de Ciências Agronômicas - UNESP; 1991. Boletim Científico vol. 2. $467 \mathrm{p}$.

Cruz-Rot M. Introdución al análisis de datos mapeados o algunas de las (muchas) cosas que puedo hacer si tengo coordenadas. Ecosistemas 2006; 15(3): 19-39.

Cunha AR, Martins D. Classificação climática para os municipios de Botucatu e São Manuel, SP. Irriga 2009; 14(1): 1-11.

Dalmaso CA, Inoue MT, Oliveira PC Fo, Marcelino VR, Pottker GS. Análise dos padrões espaciais de Ocotea odorifera (Vell.) Rohwer na Floresta Nacional de Irati (PR). Revista Ambiência 2012; 8(Especial): 559-570. http:// dx.doi.org/10.5777/ambiencia.2012.04.10.

Dalmaso CA. Padrões de interações espaciais na regeneração de Ocotea odorifera (Vell.) Rohwer na Floresta Nacional de Irati, PR [dissertação]. Irati: Programa de Pós-Graduação em Ciências Florestais, Universidade Estadual do CentroOeste; 2012.

Diggle PJ. Statistical analysis of spatial point patterns. London: Academic Press; 2003.

Figueiredo EO, Cunha RM. Levantamento das árvores com coordenadas apropriadas com GPS de alta sensibilidade. In: Figueiredo EO, Braz EM, D’Oliveira MVN, editores. Manejo de precisão em florestas tropicais: modelo digital de exploração florestal. Rio Branco: Embrapa Acre; 2007.

Fonseca RCB, Fonseca ICB. Utilização de métodos estatísticos multivariados na caracterização do mosaico sucessional em floresta semidecidual. Revista Árvore 2004; 28(3): 351-359. http://dx.doi.org/10.1590/S010067622004000300005

Fonseca RCB, Rodrigues RR. Análise estrutural e aspectos do mosaico sucessional de uma floresta semidecídua em Botucatu-SP. Scientia Forestalis 2000; 57: 27-43.

Gondim MJC. Dispersão de sementes de Trichilia spp. (Meliaceae) por aves em um fragmento de mata mesófila semidecídua, Rio Claro, SP, Brasil. Ararajuba 2001; 9(2): 101-112.

Harms KE, Wright SJ, Calderón O, Hernández A, Herre EA. Pervasive density-dependent recruitment enhances 
seedling diversity in a tropical forest. Nature 2000; 404(6777): 493-495. http://dx.doi.org/10.1038/35006630. PMid:10761916.

Instituto Brasileiro de Geografia e Estatística - IBGE. Manual técnico da vegetação brasileira. 2 ed. Rio de Janeiro: IBGE; 2012. $271 \mathrm{p}$.

Instituto de Pesquisas Jardim Botânico do Rio de Janeiro - JBRJ. Lista de espécies da flora do Brasil [online]. [acesso 2014 ago. 27]. Disponível em: http://floradobrasil.jbrj.gov.br Jordano P, Godoy JA. Frugivore-generated seed shadows: a landscape view of demographic and genetic effects. In: Levey DJ, Silva WR, Galleti M, editores. Seed dispersal and frugivory: ecology, evolution and conservation. New York: CABI Publishing; 2002. http://dx.doi. org/10.1079/9780851995250.0305.

Jorge LAB, Fonseca RCB, Arruda AA. Secondary succession dynamics of a tropical forest fragment in southeastern Brazil. In: Anais do $5^{\circ}$ Simpósio Latino-Americano Sobre Manejo Florestal; 2011; Santa Maria. Santa Maria: Universidade Federal de Santa Maria; 2011. p. 70-81.

Jorge LAB. Soil erosion fragility assessment using an impact model and geographic information system. Scientia Agricola 2009; 66(5): 658-666. http://dx.doi.org/10.1590/ S0103-90162009000500011.

Loetsch F, Zohrer F, Haller KE. Forest inventory. München: BLV Verlagsgesellchaft; 1973.469 p. vol. 2
Machado SA, Figueiredo A Fo. Dendrometria. Curitiba; 2003. 309 p.

Müeller-Dombois D, Ellemberg H. Aims and methods of vegetation ecology. New York: John Wiley \& Sons; $1974.547 \mathrm{p}$.

Ritz C, Streibig JC. Nonlinear regression with $R$. New York: Springer; 2008. 144 p.

Silva SR Fo, Engel VL. Estrutura de um fragmento de mata mesófila semidecídua secundária tardia e implicações para o manejo. In: Anais do $7^{\circ}$ Congresso Florestal Brasileiro; 1993; Curitiba. Curitiba: SBS-SBEF; 1993. vol. 1.p. 343-346.

Silvestre R, Watzlawick LF, Koehler HS, Mendonça GV, Valério AF. Florística, estrutura e distribuição espacial de espécies ocorrentes em um remanescente de floresta ombrófila mista, Castro - PR. Revista Científica Eletrônica de Engenharia Florestal 2012; 19(1): 69-86.

Viana VM, Pinheiro LAFV. Conservação da biodiversidade em fragmentos florestais. Série Técnica IPEF 1998; 12(32): 25-42.

Watzlawick LF, Gardin E, Longhi SJ. Florística, estrutura e distribuição espacial em fragmento de floresta ombrófila mista em São Francisco de Paula (RS). Revista Ambiência 2013; 9(1): 113-128. http://dx.doi.org/10.5777/ ambiencia.2013.01.08. 